\section{"Vanishing" breast implant - when a breast prosthesis is moving into the pleural cavity}

\author{
Thomas Grieser ${ }^{1 *}$, Daniel Popp ${ }^{1}$, Stephan Raab² and Thomas \\ Berghaus $^{3}$
}

'Department of Diagnostic and Interventional Radiology, University Hospital Augsburg, Augsburg, Germany

${ }^{2}$ Clinic for Cardiothoracic Surgery, Germany

${ }^{3} 1^{\text {st }}$ Medical Clinic, Department of Pneumology, Germany

\section{Case history}

A 57-year-old woman has been referred to our hospital in April 2020 because of suffering necrotizing pneumonia in the right upper lung lobe secondary to preceding pulmonary embolism half a year ago (Figure 1A,B).

The female patient was thought to have a so-called destroyed lung syndrome, but malignancy remains within the scope, however. Consequently, she has been operated on, applying an atypical partial wedge resection by means of open thoracotomy surgery in April 2020. Fortunately, final histological evaluation could exclude both lung carcinoma and metastases to the lung. In brief, destroyed lung syndrome is an end-stage destructive and suppurative lung disorder secondary to essentially infectious diseases, leading to necrotizing pneumonia [1]. Resective surgery was urgently needed because of ongoing immunotherapy due to previously known lymphatic spread of metastasizing breast carcinoma.

For this reason, it is noteworthy to mention that the female patient had had left-sided breast carcinoma about 20 years ago wherefore she underwent mastectomy on her left breast and was subsequently supplied by breast augmentations,
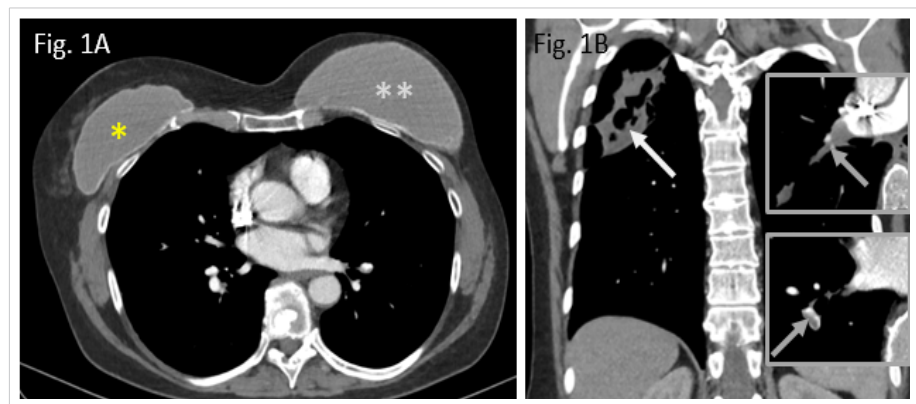

Figure 1: Preoperative situation in Nov. 2019: A: smaller breast augmentation right $\left({ }^{*}\right)$, larger breast implant left $\left({ }^{* *}\right)$; B: cavitating upper lobe pneumonia (white arrow); residual thrombi, residing in right pulmonary arteries (grey arrows in both inserts right).
More Information

*Address for Correspondence:

Thomas Grieser, MD, Clinic for Diagnostic and Interventional Radiology and Neuroradiology, University Hospital Augsburg, Stenglinstrasse 2, 86156, Augsburg, Germany,

Email: thomas.grieser@uk-augsburg.de

Submitted: July 13, 2021

Approved: August 03, 2021

Published: August 04, 2021

How to cite this article: Grieser T, Popp D Raab S, Berghaus T. "Vanishing" breast implant - when a breast prosthesis is moving into the pleural cavity. J Pulmonol Respir Res. 2021; 5: 073-075.

DOI: 10.29328/journal.jprr.1001028

Copyright: @ 2021 Grieser T, et al. This is an open access article distributed under the Creative Commons Attribution License, which permits unrestricted use, distribution, and reproduction in any medium, provided the original work is properly cited.

(D) Check for updates

OPEN ACCESS

using silicone implants on both sides (Figure 1A). On her right breast, a smaller breast implant was used for symmetry reasons as the right mammary gland has been left in place, i.e., no mastectomy was performed (here, inframammary incision technique has been applied). Size difference between both implants cannot be specified. Integrity of both implants was not assessed during the patient's stay at our hospital. Moreover, neither MRI nor ultrasonography of both breast implants were available from potentially performed previous examinations.

Furthermore, as the bilateral breast implants have been inserted in an outward clinic about 20 years ago, there were no detailed records available about type, brand, and size of the implants used. As far as it could drawn from the CT images, both breast augmentations were implanted epifascially, i.e., above the pectoralis muscles on both sides each.

After experiencing local tumour recurrence in 2016, the patient currently suffers from lymphatic spread of the disease and is continuously treated therefore.

\section{Present state}

In August 2020, the patient was re-admitted to hospital due to dyspnea and general physical weakness. Despite thorough examination including bronchoscopy with lavage procedure and bronchoscopy-guided biopsy as well as whole-body plethysmography, there was neither evidence of bronchial 
malignancy nor remaining inflammatory pulmonary changes. Laboratory tests were all found to be normal aside from a slight ventilation restriction.

In the written report on physical examination, no remarks were found about obvious chest or breast asymmetries, resp. surprisingly, no palpable abnormalities and watchable breast asymmetry could be drawn from the records.

\section{Imaging findings (CT)}

The initial computed tomography (CT) performed in November 2019 did show a rounded to oval-shaped peripheral consolidation of the right upper lung lobe (Figure 3A,B) which became more cavitated in morphology within three months, representing necrotizing pneumonia (Figure 4A,B). The destructed right upper lobe area has been removed surgically (atypical segmental resection).

Apart from postoperative residual changes within the lung tissue of the right upper lobe and adjacent pleura (Figure $5 \mathrm{~A}, \mathrm{~B})$, the more recent CT examination of the chest, dated June 2020 , however, revealed a widened $4^{\text {th }}$ right intercostal space, representing the former operative approach to right upper lobe wedge resection (Figure 2A,C). Moreover, the formerly right-sided breast implant was also missing in place (question mark in Figure 2A). Surprisingly, the same CT scan did show - more caudally - a large lobulated, though sharply delineated "soft tissue mass" in the medial costophrenic angle of patient's right thoracic cavity (asterisks in Figure 2B,D). All morphological features (configuration and density measures) share the identical implant characteristics of its own when the right implant was still positioned correctly (Figure 1A) as well as they are morphologically identical when compared with those of the left-sided breast implant (situation after mastectomy). Thus, "soft tissue mass" in the right costophrenic angle therefore proves to be the interpleural dislocated ipsilateral breast implant.

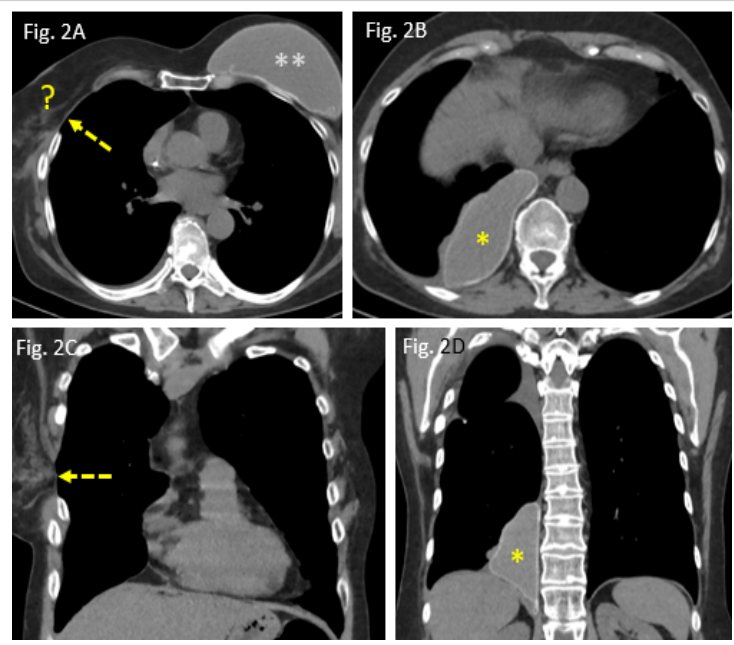

Figure 2: Postoperative situation in June 2020: A: widened intercostal space after thoracotomy (dashed arrow); right breast implant missed (?); B: migrated implant, located in the posteromedial costophrenic angle $\left(^{*}\right)$; C/D: coronal CT reformats showing the intercostal defect (dashed arrow), allowing the implant to move intrapleurally $\left({ }^{*}\right)$.

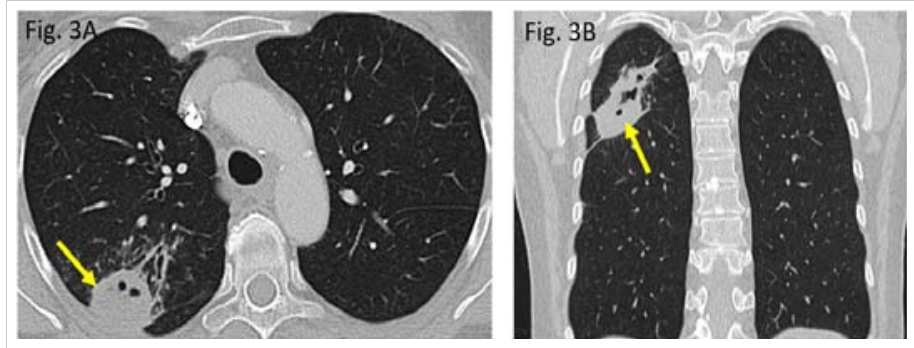

Figure 3: Preoperative pulmonary situation in November 2019: A/B: a rounded peripheral consolidation in the right upper lobe posterior segment $\mathrm{S} 2$ (arrow), showing small pneumatoceles.

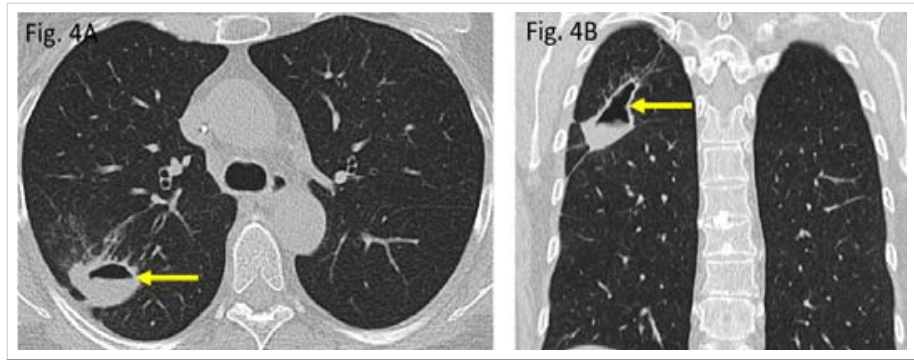

Figure 4: Follow-up CT scan in February 2020: A/B: lung consolidation is shrinking while is rendering liquid (air-fluid-level) thus, becoming cavernated (arrows).

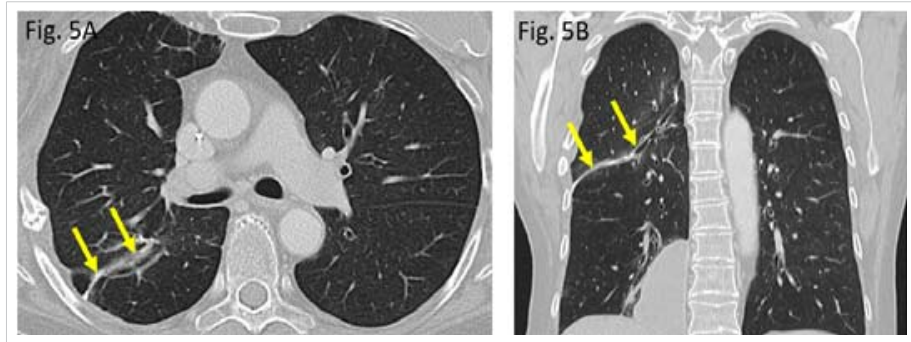

Figure 5: Postoperative pulmonary situation in August 2020: A/B: a small, curvilinear suture across the upper lobe, indicating atypical wedge resection in the right upper lobe (arrows)

\section{Discussion}

This case shown here represents a rare situation where the breast implant is spontaneously and inadvertently migrated from its submammary position via the thoracic wall into the ipsilateral pleural cavity after performing an ipsilateral thoracotomy due to atypical wedge resection of the right upper lobe four months ago. Intraoperatively, the implant has been neither dislodged nor manipulated in any way.

In the literature, there are some sparse case descriptions where such breast implant migrations are encountered after VATS procedure (video-assisted thoracoscopy) [2] and open thoracotomy surgery [3]. Interestingly, our case report is quite similar to those which was published by Dutch colleagues in 2014 [4].

Considering the etiology and pathomechanism of such an implant migration as shown here, there is a common agreement that both a leakage of the implant's fibrous capsule and an operative transection of the intercostal thoracic wall are prerequisite to create a potential migrating pathway to allow implants moving towards the pleural cavity [5]. 
Additionally, it is believed that the negative pressure within the pleural cavity also alleviates the unidirectional herniation by "sucking in the implant" into the interpleural space [6]. Sometimes, external repetitive pressures such as stretching massages may cause or trigger such an implant dislocation. Furthermore, there are cases described in which, seemingly, implant migration does occur without known preceding thoracic surgery [7]. Eventually, there are cases published in the literature with intrapleural spreading of disrupted breast implant debris [8].

With our patient, thanks to the absence of any discomfort or pain, it was concluded after agreed statement of an interdisciplinary round table discussion not to remove the dislocated implant surgically because of potential intercostal tissue damage and subsequent pain to await. More astonishing, the clinicians involved in this case wondered the fact that the missed implant of her right breast remained either unnoticed or has been completely neglected by the female patient.

In this short communication, we present a rare and unusual case of an obviously vanishing breast implant which is found to be inadvertently migrated into the adjacent pleural space after undergoing thoracic surgery.

According to common legal policy at our institution, an approval for case reports is generally provided as it was obtained in this particular case.

\section{References}

1. Tsai $\mathrm{YF}, \mathrm{Ku} \mathrm{YH}$. Necrotizing pneumonia: a rare complication of pneumonia requiring special consideration. Curr Opin Pulm Med. 2012; 18: 246-252.

PubMed: https://pubmed.ncbi.nlm.nih.gov/22388585/

2. Lehoux JM, Tchantchaleishvili V, Jones CE. Intrathoracic migration of a silicone breast implant after video-assisted thoracoscopic surgery. Ann Thorac Surg. 2013; 96: 326.

PubMed: https://pubmed.ncbi.nlm.nih.gov/23816091/

3. Mehta AM, Bard MPL, van Straten A, van Beijeren I, Rijna H Intrathoracic migration of a breast prosthesis after thoracotomy. J Thorac Cardiovasc Surg. 2008; 135: 206-207. PubMed: https://pubmed.ncbi.nlm.nih.gov/18179945/

4. Bruintjes M, Schouten C, Fabré J, an den Wildenberg FJH. Where the PIP is the implant? J Plastic Reconstr Aesthet Surg. 2014; 67: 1148-1150.

5. Kim H, Heo C, Baek R, Minn K, Kim S, et al. Breast implant migration into pleural cavity. J Plastic Reconstr Aesthet Surg. 2009; 62: e89-e90. PubMed: https://pubmed.ncbi.nlm.nih.gov/19081310/

6. Sykes JB, Rosella PA. Intrathoracic migration of a silicone breast implant 5 months after video-assisted thoracoscopic surgery. J Comput Assist Tomogr. 2012; 36: 306-307.

PubMed: https://pubmed.ncbi.nIm.nih.gov/22592613/

7. Chen ZY, Wang ZG, Kuang RX, et al. Implant found in thoracic cavity after breast augmentation. Plast Reconstr Surg. 2008; 116: 1826-1827. PubMed: https://pubmed.ncbi.nlm.nih.gov/16267478/

8. Lee JY, Kim HK, Kim WS, Park BY, Bae TH, et al. Rupture and intrapleural migration of a cohesive silicone gel implant after augmentation mammoplasty: a case report. J Korean Soc Plast Reconstr Surg. 2011; 38: $323-325$ 